\title{
Éditorial
}

\section{LES TECHNOLOGIES ÉMERGENTES EN CONTEXTE EXTRÊME : DE L'ADAPTATION À L'ANTICIPATION ?}

\author{
Cécile Godé, François de Corbière, Jessie Pallud
}

ESKA | «Systèmes d'information \& management »

2020/2 Volume 25 | pages 3 à 6

ISSN 1260-4984

ISBN 9782747230728

Article disponible en ligne à l'adresse :

https://www.cairn.info/revue-systemes-d-information-etmanagement-2020-2-page-3.htm

Distribution électronique Cairn.info pour ESKA.

(c) ESKA. Tous droits réservés pour tous pays.

La reproduction ou représentation de cet article, notamment par photocopie, n'est autorisée que dans les limites des conditions générales d'utilisation du site ou, le cas échéant, des conditions générales de la licence souscrite par votre établissement. Toute autre reproduction ou représentation, en tout ou partie, sous quelque forme et de quelque manière que ce soit, est interdite sauf accord préalable et écrit de l'éditeur, en dehors des cas prévus par la législation en vigueur en France. Il est précisé que son stockage dans une base de données est également interdit. 


\title{
Éditorial
}

\section{Les technologies émergentes en contexte extrême : de l'adaptation à l'anticipation?}

\author{
Cécile Godé ${ }^{1}$, François de Corbière $e^{2}$ EJessie Pallud ${ }^{3}$
}

À l'heure où nous écrivons ces lignes, la France entame sa septième semaine de confinement, tactique majeure dans sa stratégie de combat contre la pandémie de Covid-19. Alors que le télétravail ne concernait jusqu'ici que $30 \%$ des salariés du secteur privé (Malakoff Humanis, 2020), il est ces dernières semaines devenu la norme pour la très large majorité des Français confinés. D'ici 2024, les réunions en présentiel ne représenteront plus que $25 \%$ des réunions d'entreprise, contre $60 \%$ avant la crise du Covid-19 (Gartner, 2020). Cette tendance pourrait d'ailleurs modifier l'effet millefeuille généralement observé au sein des entreprises où les outils de communication ne se substituent pas aux réunions en face à face (Boukef Charki et Kalika, 2006). Parallèlement, les salariés des secteurs « indispensables au fonctionnement du pays » (médical et paramédical, énergie, propreté, agriculture, transport, distribution, etc.) sont appelés à se rendre physiquement sur leur lieu de travail (allocution de Bruno Le Maire, Ministre de l'Économie et des Finances, mercredi 18 mars 2020).
Qu'il s'agisse de travail à distance ou en présentiel, la crise sanitaire du Covid-19 bouleverse considérablement le fonctionnement des organisations et des personnels. Elle les plonge en contexte extrême (Hällgren et al., 2018; Godé, 2015), les confrontant à un haut niveau d'incertitude quant au futur proche, à des changements d'envergure dans leur quotidien et à des risques sanitaires et vitaux importants. Chacun fait face à cette situation inédite et imprévue (Weick et Sutcliffe, 2007) en délaissant ou ajustant les modes et standards de travail habituels pour mettre en œuvre des processus opérationnels et de répartition des tâches adaptés aux défis à relever (Godé et al., 2016). Ainsi, face à la pénurie soudaine de gel hydroalcoolique, le groupe LVMH a demandé à sa branche parfums et cosmétiques (dont Guerlain, Dior et Givenchy parmi les plus fameux) de réorganiser en quelques jours sa chaîne de production pour être en mesure de produire plusieurs dizaines de milliers de litres hebdomadaires à destination des hôpitaux de Paris.

Dans ce contexte extrême, la question du rôle joué par les systèmes d'information et

\footnotetext{
${ }^{1}$ Aix Marseille Univ, CRET-LOG, Aix-en-Provence, France

${ }^{2}$ IMT Atlantique, LEMNA, Nantes, France

${ }^{3}$ EM Strasbourg Business School, Université de Strasbourg, HuManiS, France
} 
les technologies qui les composent se pose plus que jamais. Comme l'affirmait Lebraty dès 2013 dans son éditorial, « les SI offrent les conditions permettant de faire face à ces situations [extrêmes] » (p. 7). L'observe-t-on aujourd'hui ? La réponse est en demi-teinte.

Oui, les technologies viennent massivement et efficacement en support de nos capacités d'adaptation. C'est particulièrement le cas des technologies dites émergentes, ces « technologies nouvelles en forte croissance, caractérisées par un certain degré de cohérence et de persistance [dont] les impacts les plus significatifs sont encore en émergence, demeurant incertains et ambigus » (Rotolo et al., 2015, p. 1831). Durant la crise Covid-19, les systèmes basés sur l'intelligence artificielle (traitement des données massives, systèmes de tracking, robots intelligents, etc.), l'impression 3D et les outils d'apprentissage et de communication digitaux (réalité virtuelle, réalité augmentée, plateforme de visioconférence, etc.) occupent le devant de la scène. Par exemple, en Chine le robot domestique Little Peanut, associé au réseau social WeChat, remplace le personnel pour distribuer les repas dans des hôtels où sont confinées des personnes infectées par le virus. Lorsqu'en France, la téléconsultation via des plateformes en ligne est devenue la norme entre les médecins et les patients symptomatiques, certains médecins chinois utilisent depuis peu la réalité virtuelle couplée à la $5 \mathrm{G}$ pour « visiter » dans leur chambre d'hôpital les patients infectés. Des institutions de formation supérieure françaises (écoles de commerce et d'ingénieurs notamment) ont pris la décision de dématérialiser leurs journées portes ouvertes ; plus complexes, certaines dématérialisent également leurs concours d'entrée en utilisant des technologies antifraudes, de la vidéo et un algorithme d'intelligence artificielle de surveillance censé traiter les données en temps réel.

Comme l'illustrent ces quelques exemples, les technologies émergentes offrent un large éventail de fonctionnalités venant en appui des capacités d'adaptation des entreprises et des personnels, nécessaires durant cette crise sanitaire. Pour autant, est-ce suffisant? S'adapter implique d'adopter a posteriori des pratiques et des process en accord avec des situations et des contraintes nouvelles, que celles-ci soient temporaires ou pérennes. L'adaptation est une réaction face à l'imprévu, permettant de garantir la continuité de l'activité. Mais qu'en est-il des capacités d'anticipation face au Covid-19? Les technologies émergentes sont-elles, là aussi, au rendez-vous ?

L'intelligence artificielle est dans ce domaine pleine de promesses, mais force est de relativiser sa valeur prédictive sur cette pandémie. En effet, les systèmes de prédiction sont en cours de développement et se révèlent aujourd'hui insuffisamment matures pour, par exemple, anticiper une crise sanitaire telle que nous la traversons, fournir des modélisations robustes et fiables sur lesquelles les organisations pourraient s'appuyer pour préparer le futur ou encore prédire l'évolution du virus. La qualité et la fiabilité des données, qui viennent nourrir ces systèmes, sont également à questionner, lorsque la hauteur des enjeux et la pression temporelle sur les résultats jouent à plein.

Pourtant, BlueDot, une start-up canadienne spécialisée dans la détection et le suivi de maladies infectieuses, alertait dès fin décembre sur les risques d'une épidémie. Grâce à un algorithme capable d'analyser, toutes les 15 minutes, des centaines de milliers d'articles, de rapports, de forums professionnels et de les croiser avec des données climatiques, sanitaires, de transport, etc., l'intelligence artificielle peut reconnaitre les signes d'une épidémie ou d'une pandémie et en informer ses clients (institutionnels et privés). Mais, comme le précise le fondateur de BlueDot, Kamran Khan « les données faisaient écho à l'épidémie de SARS d'il y a 17 ans, nous ne savions pas à quel point ce virus serait contagieux» (AFP, le 26 mars 2020à 06h45). BlueDot a donc prédit une épidémie, comme il y en a 
tant chaque année dans de nombreux pays ; la start-up était loin de modéliser la pandémie que nous connaissons aujourd'hui. Le MIT utilise également l'intelligence artificielle pour prédire la propagation du Covid-19 et mesurer notamment l'effet de la distanciation physique et des mesures de confinement sur son évolution. À partir d'une évaluation a posteriori, il apparaît que les résultats obtenus au 1er avril 2020 à partir d'une modélisation des données collectées en mars sont proches de la réalité. Pour autant, l'outil est encore trop «jeune» pour garantir la fiabilité des prédictions à venir. Une autre illustration renvoie à l'intelligence artificielle utilisée pour prédire les structures des protéines du Covid-19. L'entreprise britannique DeepMind (Google) a, par exemple, développé un système d'intelligence artificielle nommé AlphaFold produisant des modélisations des structures protéinaires du virus et les partageant en libre accès. Bien qu'il puisse « contribuer à l'interrogation de la communauté scientifique sur le fonctionnement du virus, et servir de plateforme pour établir des pistes de futurs travaux expérimentaux visant à développer des traitements », AlphaFold reste à un stade de développement expérimental qui peut légitimement conduire à interroger la robustesse de ses résultats. Enfin, on voit aussi émerger des applications mobiles qui tracent nos déplacements et nos contacts sociaux pour isoler les personnes contaminées et tester celles qui ont été à leur contact. Cette solution, prônée par la Commission Européenne, pose des questions juridiques - celle par exemple de son respect du Règlement Général sur la Protection des Données (RGPD), sujet qui sera prochainement débattu à l'Assemblée Nationale - mais interroge également quant à son efficacité à venir face à une population qui ne l'adopte pas massivement (l'application permettra d'obtenir des résultats fiables si, et uniquement si, une majorité de la population l'utilise).

Les systèmes d'information et les technologies permettent donc de faire face aux contextes extrêmes, ou tout du moins à celui que nous traversons actuellement. Ils soutiennent efficacement les capacités d'adaptation des organisations et des hommes, et développent des capacités d'anticipation, certes encore au stade expérimental, mais prometteuses. En contexte extrême, la pression du temps est intense, il faut trouver des solutions rapidement. Les systèmes prédictifs d'intelligence artificielle y gagnent sans aucun doute, bénéficiant d'un engouement et d'un investissement vertigineux qui laissent à penser une amélioration rapide et significative de la qualité des modèles produits demain et de la fiabilité de leurs résultats. Reste centrale la problématique de la qualité de l'information, et ce à travers plusieurs dimensions (de Corbière, 2011). Sous contrainte de temps et d'urgence d'adaptation, nos organisations souffrent de la qualité des données qui alimentent les systèmes d'information, ainsi que de la qualité de l'information produite. Pour la gestion quotidienne des affaires et des opérations, la multiplication des outils de partage, de stockage et de communication rend l'information volatile et non consolidée, dont l'unicité et la mise à jour sont très difficiles à garantir.

Dans ce numéro, les trois articles, rédigés en langue anglaise, abordent des thématiques particulièrement pertinentes dans les circonstances actuelles.

Le premier, coécrit par Samuel FossoWamba, Shahriar Akter et Cameron Guthrie, s'intéresse à la mobilisation du big data analytics (BDA) sur la performance des entreprises. Sur labase d'un modèle quantitatif et d'un questionnaire administré auprès de 202 entreprises, leurs résultats confirment que l'agilité organisationnelle contribue fortement à attirer des investissements dans le BDA en vue de créer une valeur commerciale stratégique et d'améliorer la performance des entreprises. La capacité d'agilité organisationnelle pour gérer les affaires et les opérations est au centre des préoccupations 
actuelles. L'analyse proposée par les auteurs est en cela particulièrement pertinente, ouvrant la voie à un approfondissement de leurs résultats en période de confinement, durant laquelle agilité a souvent été synonyme de survie pour de nombreuses organisations.

Dans le second article, Isabelle Walsh étudie les relations entre la culture numérique des utilisateurs et les différentes facettes de leur usage des TI, ainsi que l'émergence et le développement de besoins numériques. À partir d'une recherche mixte exploitant des données quantitatives et qualitatives dans les perspectives variance et process, l'auteure révèle l'existence de boucles causales entre les construits et explique le processus d' «acculturation numérique». Un processus d'acculturation numérique qui s'est certainement accéléré ces dernières semaines pour nombre d'entre nous, soudainement et durablement confronté au télétravail.

Enfin, Simon Bourdeau, Marie-Claude Petit et Sylvain Goyette proposent un article pédagogique sur le développement de compétences associées à la gestion de projet en technologies de l'information, et notamment à la réalisation d'estimations en termes de fonctionnalités et de calendrier. À partir d'observations et de données recueillies auprès de 123 étudiants ayant participé à un cours de gestion de projet TI, les auteurs présentent les avantages de la formation par simulation en utilisant des briques LEGO® afin de développer et tester une simulation en estimation de projets. La simulation, que ce soit en présentiel ou en environnement virtuel ou augmenté, est sans conteste une voie prometteuse pour développer les compétences d'adaptation dont les entreprises ont besoin pour accroître leur résilience face à l'imprévu. Ainsi, dans la période que nous vivons où la gestion de l'urgence est devenue la norme sans que nous n'y soyons jamais vraiment préparés, cet article qui valorise la simulation semble riche d'enseignements pratiques à destination des organisations et des institutions supérieures de formation.

\section{BIBLIOGRAPHIE}

Boukef Charki N., Kalika M. (2006), «La théorie du millefeuille, le rôle du contexte ", Systèmes d'Information et Management, vol. 11, n4, p. 29-54.

de Corbière F. (2011), "SIIO et amélioration de la qualité des données : analyse de la synchronisation des données par catalogues électroniques ", Systèmes d'Information et Management, vol. 16, n³, p. 45-79.

Hällgren M., Rouleau L., de Rond M. (2018), "A matter of life or death: How extreme context research matters for management and organization studies", The Academy of Management Annals, vol. 12, n¹, p. 111-153.

Gartner, Press releases, June 2nd, 2020, accessed on June 8th 2020, https://www.gartner.com/en/ newsroom/press-releases/2020-06-02-gartnersays-worldwide-end-user-spending-on-cloudbased-web-conferencing-solutions-will-grownearly-25-percent-in-2020

Godé C. (2015), La coordination des équipes en environnement extrême : pratiques de travail et usages technologiques en situation d'incertitude, ISTE Edition, Paris, 9781784051143 , $148 \mathrm{p}$.

Godé C., Melkonian T., Picq T. (2016), « Performance collective : quels enseignements des contextes extrêmes ? ", Revue Française de Gestion, vol. 42, n²57, p. 73-78.

Lebraty J-F. (2013), « Editorial. SI et situations extrêmes ", Systèmes d'Information et Management, vol. $18, \mathrm{n}^{\circ} 1$, p. 3-10.

Malakoff Humanis (2020), Etude Télétravail 2020 : Regards croisés Salariés / Entreprises, $3^{\mathrm{e}}$ édition, mars, https://newsroom.malakoffhumanis.com/assets/synthese-etude-teletravail-2020-2a13-63a59.html?lang $=$ fr

Rotolo D., Hicks D., Martin B.R. (2015), "What is an emerging technology?", Research Policy, vol. $44, n^{\circ} 10$, p. $1827-1843$.

Weick K., Sutcliffe K. (2007), Managing the unexpected: Resilient performance in an age of uncertainty, John Wiley \& Sons, New York. 